Strabismus 0927-3972/94/US\$3.50

Strabismus-1994, Vol. 2, No. 3,

pp. $143-146$

(C) Aolus Press

Buren (The Netherlands) 1994

Accepted I August I994

\section{Facilitation of vertical vergence by horizontal saccades, found in a patient with dissociated vertical deviation}

\author{
Huibert Jan Simonsz ${ }^{1}$ \\ Laurentius Johannes van Rijn ${ }^{2}$
}

1 Orthoptics \& Neuro-ophthaimology, Ophthalmology, University Hospital Dijkzigt

${ }^{2}$ Department of Physiology I, Erasmus University, Rotterdam, The Netherlands

\begin{abstract}
The authors examined vertical vergence in a 15-year-old girl with dissociated vertical deviation, a $6^{\circ}$ convergent strabismus, no binocular vision, latent nystagmus, and a minimal left amblyopia. Eye movements were recorded during $4 \mathrm{~s}$-periods of ( 1 ) both eyes open, alternated with $4 \mathrm{~s}$ periods of (2a) right eye covered, (2b) left eye covered or (2c) both eyes closed. The patient preferred fixation with the right eye; when this eye was covered, the left eye took over fixation with an almost completely monocular, downward saccade (a horizontal saccade occurred at the same time). The right eye only made a very small saccade, and then started to drift upward with an exponentially decreasing velocity. Enright has shown that fast convergence or divergence may occur when a horizontal saccade is performed at the same time. Similarly, the authors suspected that, in their patient, vertical vergence was facilitated by the horizontal saccade that resulted, in this case of horizontal strabismus, from alternation of fixation. To examine whether this was the case, they compensated for the horizontal strabismus by placing a 30 prism-dioptre base-out prism in front of the left eye (the patient had no binocular vision). With the prism in place, the horizontal saccade that occurred when the right eye was covered was smaller, and only little vertical vergence occurred together with the horizontal saccade. It seems possible that vertical vergence is also facilitated by horizontal saccades.
\end{abstract}

Key words Eye movements; strabismus; esotropia; dissociated vertical deviation; saccades; convergence

Introduction Convergence and divergence are slow eye movements, not exceeding $20 \% \mathrm{~s}$ in velocity. In I984, Enright ${ }^{1}$ reported that convergence or divergence can be accelerated several times by performing a saccade, a fast eye movement, at the same time. This result conforms to vision under natural circumstances: Vision during everyday life demands that we are able to look quickly from a near object to a distant object, or vice versa. The two,
Correspondence to: PD Dr. H.J. Simonsz, Orthoptics \& Neuro-ophthalmology, Afdeling Oogheelkunde, Academisch Ziekenhuis Dijkzigt, Dr. Molewaterplein 40, NL 3015 GD Rotterdam, The Netherlands, fax: 00.3I.10.4635 I05 
Fig. I. Vertical eye position (top two traces) and horizontal eye position (bottom two traces) of both eyes before (left), during (middle) and after (right) 4 s cover of the right eye. Abscissa: time, horizontal bar equals I.5 seconds. Vertical bar equals $20^{\circ}$. When the right eye was covered (the patient preferred fixation with the right eye), the left eye (continuous line) took over fixation with a downward, almost completely monocular saccade. The right eye (interrupted line) only made a very small downward saccade, after which a slow upward movement, typical of dissociated vertical deviation, started. This had an exponentially decreasing velocity with a time constant of about Is. When the cover was lifted $4 \mathrm{~s}$ later, the right eye immediately retook fixation, also with an almost completely monocular saccade. In addition to the DVD, the patient had a convergent strabismus and latent nystagmus. Hence, covering the right eye also resulted in a horizontal saccade (lower two traces). Note the latent nystagmus, made manifest by unilateral cover. near and distant, objects are usually at different places in the visual field of the observer, so under natural circumstances a fast convergence or fast divergence is only needed when performing a saccade at the same time. In addition, Enright noted that vertical saccades are nearly as effective as horizontal saccades in mediating a change in horizontal vergence. As part of a larger study, we examined several patients with dissociated vertical deviation to describe the exact vertical and torsional components of the dissociated vertical eye movements in these patients (van Rijn \& Simonsz, submitted), and found one patient who had very fast vertical vergence when performing a horizontal saccade at the same time. This patient was the subject of this study.

Methods The patient was seated at $143 \mathrm{~cm}$ distance from a Snellen letter chart that was brightly illuminated ( $1000 \mathrm{~cd} / \mathrm{m}^{2}$ ). The subject was asked to fixate the one particular optotype that was located straight ahead. The elements of this optotype subtended 5 arc min of visual angle. A shutter device just in front of the subject could be used to occlude either one of both eyes. The experiment consisted of either 9 or I 8 measurements of I 2 s each. Eye movements were recorded during $4 \mathrm{~s}$-periods of ( $\mathrm{I}$ ) both eyes open, alternated with $4 \mathrm{~s}$-periods of (2a) right eye covered, (2b) left eye covered or (2c) both eyes closed. Horizontal, vertical and torsional components of eye movements were registered of both eyes simultaneously, with scleral induction coils (Skalar). The noise level of this technique was less than $0 . \mathrm{I}^{\circ}$ and the linearity better than 0.8 . Eye position signals were amplified, filtered (cut-off frequency: I $25 \mathrm{~Hz}$ ), and sampled at $250 \mathrm{~Hz}$. Samples were stored on a PDP I I/73 minicomputer for off-line analysis. After adequate correction procedures for coil-misalignment all data were plotted.

The patient was a 15 -year-old girl with a congenital (or infantile) strabismus syndrome: she had a manifest $6^{\circ}$ convergent strabismus that increased to $15^{\circ}$ when covering one eye, no binocular vision, a latent nystagmus, a dissociated vertical deviation and a minimal left amblyopia.

Results When the right eye (the patient preferred fixation with the right eye because the left eye had a minimal amblyopia) was covered, the left eye took over fixation with an almost completely monocular, downward saccade

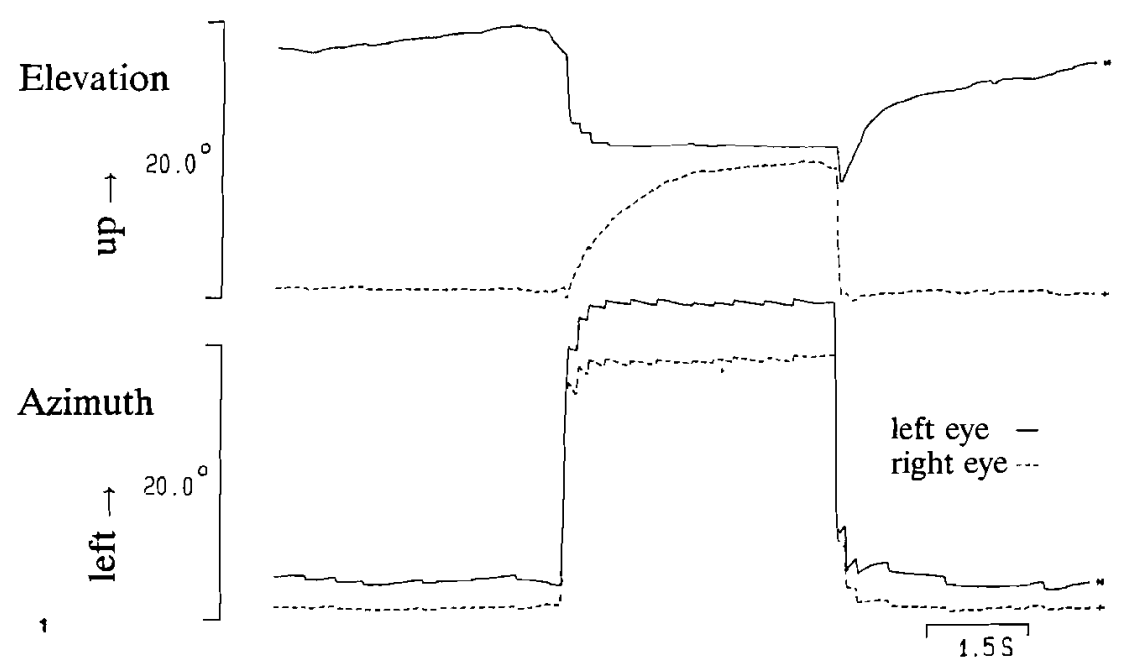



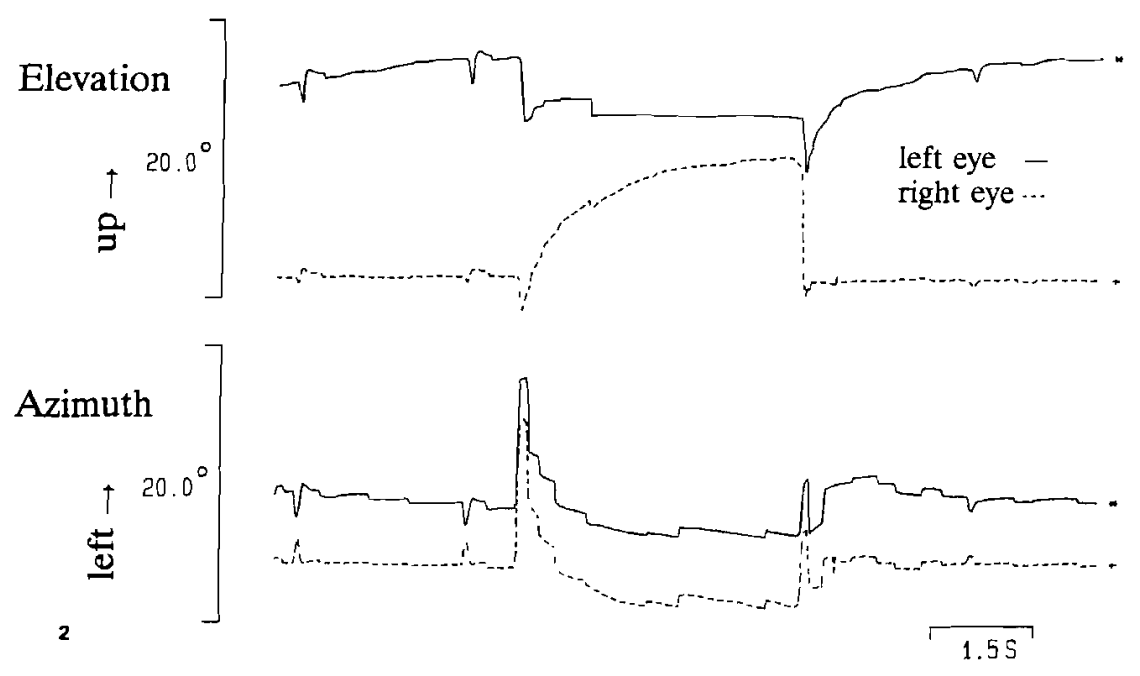

(Fig. I). The right eye only made a very small downward saccade, and then started to drift upward with an exponentially decreasing velocity, with a time constant of about one second. Initially we were puzzled by the finding and thought of several hypotheses to explain it. One of the hypotheses was that vertical vergence was facilitated by the horizontal saccade that, in this case of horizontal strabismus, resulted from alternation of fixation. To examine whether this was the case, we compensated for the horizontal strabismus by placing a 30 prism-dioptre base-out prism in front of the left eye (note that the patient did not have binocular vision). The patient no longer needed to perform the $\pm 15^{\circ}$ horizontal saccade to take over fixation with the left eye, but she still persisted in performing a small saccade, because she subconsciously still expected the target to be at a different place when fixation was alternated (Fig. 2). Nevertheless, with the prism in place the initial horizontal saccade was smaller. Accordingly, the vertical downward saccade was much more conjugate; very little vertical vergence occurred together with the horizontal saccade. In other words, fast vergence was much less facilitated by the horizontal saccade.

Discussion It seems likely that, in this patient, the horizontal saccade enabled fast vertical vergence. $Z_{\text {Zubcov }}{ }^{2}$ also recently reported on asymmetric vertical saccades occurring in patients with dissociated vertical deviation. At first we thought that monocular downward saccade was a specific phenomenon occurring in patients with dissociated vertical deviation; a puzzling hypothesis, for why would the riMLF issue a purely unilateral command? Then we noticed, however, that these unilateral downward saccades occurred only when a horizontal eye movement occurred simultaneously and we sought ways to eliminate the horizontal component. We did not succeed fully in this, because alternating fixation in a strabismus patient will invariably lead to an eye movement, as the brain expects to see the target at a different location. By putting a prism, equal in strength to the latent angle of strabismus, in front of one eye, this eye movement is no longer necessary. (This is probably noticed immediately before the saccade starts).

The saccade performed was smaller than before, and was followed immediately by a counter-saccade. In our patient, the amount of fast vergence occurring during this smaller horizontal saccade was much smaller than before
Fig. 2. Vertical eye position of both eyes before, during and after $4 \mathrm{~s}$ cover of the right eye. We compensated for the horizontal strabismus by placing a 30 prism-dioptre base-out prism in front of the left eye that compensated for the (latent) objective angle of strabismus. The patient no longer needed to perform the horizontal saccade to take over fixation with the left eye, but she still persisted in performing a small saccade, because she subconsciously still expected the target to be at a different place when fixation was alternated. The initial horizontal saccade was smaller, however, and the vertical downward saccade was much more conjugate: very little fast vertical vergence occurred together with the horizontal saccade. 
curring during this smaller horizontal saccade was much smaller than before putting the prism before one eye. This finding is compatible with the idea that also vertical vergence can be facilitated by horizontal saccades.

The issue of what causes facilitation of vergence by saccades has not been definitely settled, but an interesting explanation has been forwarded by Fitzgibbon et al. ${ }^{3}$ The convergence- (and some divergence-) burst cells in the mesencephalic reticular formation dorsolateral of the oculomotor nucleus are connected to the oculomotor nuclei via burst-tonic cells. Some control over the convergence burst cells is exerted by the omnipause cells in the brain stem, that fire continuously during pauses between saccades. Fitzgibbon et al. surmised that facilitation of horizontal vergence occurs because the omnipause neurons stop firing when a saccade begins. It remains to be seen whether facilitation of vertical vergence by horizontal saccades, as demonstrated in our patient, is mediated by the same mechanism.

\section{References}

I Enright JT, Changes in vergence mediated by saccades. J Physiol I984; 350:9-3I.

2 Zubcov AA, Goldstein HP, Reinecke RD. Dissociated vertical deviation (DVD): The saccadic and slow eye movements. Strabismus I 994; 2:I - I I.

3 FitzGibbon EJ, Zee DS, Optican LM. A model of saccade-vergence interaction. Invest Ophthaimol I992; 33(4,ARVO Suppl): I IOI. 